\title{
Bridging semantic gap between high-level and low-level features in content-based video retrieval using multi-stage ESN-SVM classifier
}

\author{
N BRINDHA* and P VISALAKSHI \\ PSG College of Technology, Coimbatore 641004, India \\ e-mail: brindhacsepsgtech@gmail.com; spv@ece.psgtech.ac.in
}

MS received 2 October 2015; revised 25 April 2016; accepted 22 July 2016

\begin{abstract}
Content-based video retrieval system aims at assisting a user to retrieve targeted video sequence in a large database. Most of the search engines use textual annotations to retrieve videos. These types of engines offer a low-level abstraction while the user seeks high-level semantics. Bridging this type of semantic gap in video retrieval remains an important challenge. In this paper, colour, texture and shapes are considered to be low-level features and motion is a high-level feature. Colour histograms convert the RGB colour space into $\mathrm{YcbCr}$ and extract hue and saturation values from frames. After colour extraction, filter mask is applied and gradient value is computed. Gradient and threshold values are compared to draw the edge map. Edges are smoothed for sharpening to remove the unnecessary connected components. These diverse shapes are then extracted and stored in shape feature vectors. Finally, an SVM classifier is used for classification of low-level features. For high-level features, depth images are extracted for motion feature identification and classification is done via echo state neural networks (ESN). ESN are a supervised learning technique and follow the principle of recurrent neural networks. ESN are well known for time series classification and also proved their effective performance in gesture detection. By combining the existing algorithms, a high-performance multimedia event detection system is constructed. The effectiveness and efficiency of proposed event detection mechanism is validated using MSR 3D action pair dataset. Experimental results show that the detection accuracy of proposed combination is better than those of other algorithms
\end{abstract}

Keywords. Classification; feature selection; SVM; ESN; spatio-temporal structure.

\section{Introduction}

Advancement in technology and emergence of social media led to capturing, storing and broadcasting digital video through web technologies. For example the number of videos uploaded per minute reached $72 \mathrm{~h}$ in Youtube. Searching videos in web has become a challenging task due to the fact that search engines use Meta data (i.e., textual annotations) to index and retrieve the results for the query. Enhancement of video retrieval process is needed in traditional search engines to explore rich media contents [1]. Content-based video retrieval technique (CBVR) is used in retrieval of a similar video from video database. It is an extension of content-based image retrieval (CBIR) systems. Features such as colour, shape, texture, etc. are considered for images. On the other hand video retrieval process also follows the same system on feature set obtained in frames and some temporal analysis like shot detection to group frames and select better key frames accordingly. In addition to the video frames the dataset may also contain audio, text,

*For correspondence etc. For event detection, motion-based and object-based features are needed.

Video analysis is done in two phases: low-level visual concepts such as colour, shapes, textures, etc., and highlevel features such as objects and events. Concepts refer to a specific instance or actions. Events in a video can be used to depict significant information. The events can be a collection of objects, motion and scenes. For example a fishing event consists of concepts like fish, people and fish rod and actions like landing of fish [2]. Extraction of features at the two levels is different. Semantic content extraction is a complex process since it requires user interaction and domain-specific knowledge. However, visual content is extracted automatically and it is independent of domains [3]. Various methods have been proposed for identifying events in videos, including sports [4] and surveillance videos [5]. These studies have focused on using spatiotemporal structures of events. Lu et al [3] used HMM to classify three types of highlights with the help of players location feature and constant camera motion. Adam et al [5] have used a crowd of people in surveillance video from optical flows to identify the unusual events, for example, running in the mall. 
These methods are applicable when the target videos have a spatio-temporal structure, i.e., when the target events are specified. Our aim is to detect an event with certain lowlevel features; an attempt of this work has been made on the Microsoft Research (MSR) 3D action pair dataset. Frames obtained from the MSR dataset are given in figure 1. Earlier works have identified various strategies that are employed for the event detection mechanism. The basic concept of video retrieval consists of three steps: key frames, feature extraction and matching. Initially videos are divided into frames; then corresponding features from frames are extracted. Finally the features are matched with training sets. A methodology is proposed by converting videos into frames and segmenting frames as objects in the form of images. Feature extraction is done via SIFT algorithm and matching is done through nearest neighbour search [6]. An event detection is made in crowded videos with partial occlusion and background clutter. This is done in three stages: volumetric representation against oversegmented spatio-temporal video volumes, representing shape-based features using a flow and treating events as an atomic entity, i.e., matching is done through space and time [7]. A joint framework for intermediate representation of video features from event- and concept-based videos has been proposed. Classifier interference and latent intermediate representation are tightly correlated such that the classifier depends on both the task and specific classifier. Certain other works have concentrated on the popular Bag-of-Words framework [8]. In this paper, a model is proposed to bridge the semantic gap between low-level and high-level features by extracting colour, texture and shape feature using colour histogram and edge detection methods and SVM for classification. The result of classification is used as a model for second-stage classifier such that it selects frames that are labelled as relational to the query image. This is done via SVM vectors that identify frames. These frames are then subjected to deep learning for motion detection and ESN algorithm is used for the final process of result classification. With the help of a multi-stage classifier it is proved that the proposed combination reduces false positive rate and detection accuracy is increased. Remaining of this paper is structured as follows: section 2 gives an overview of the CBVR method, section 3 presents our proposed methodology and experimental set-up and dataset description is given in section 4 ; section 5 gives the results and discussion and this is followed by conclusion in section 6.
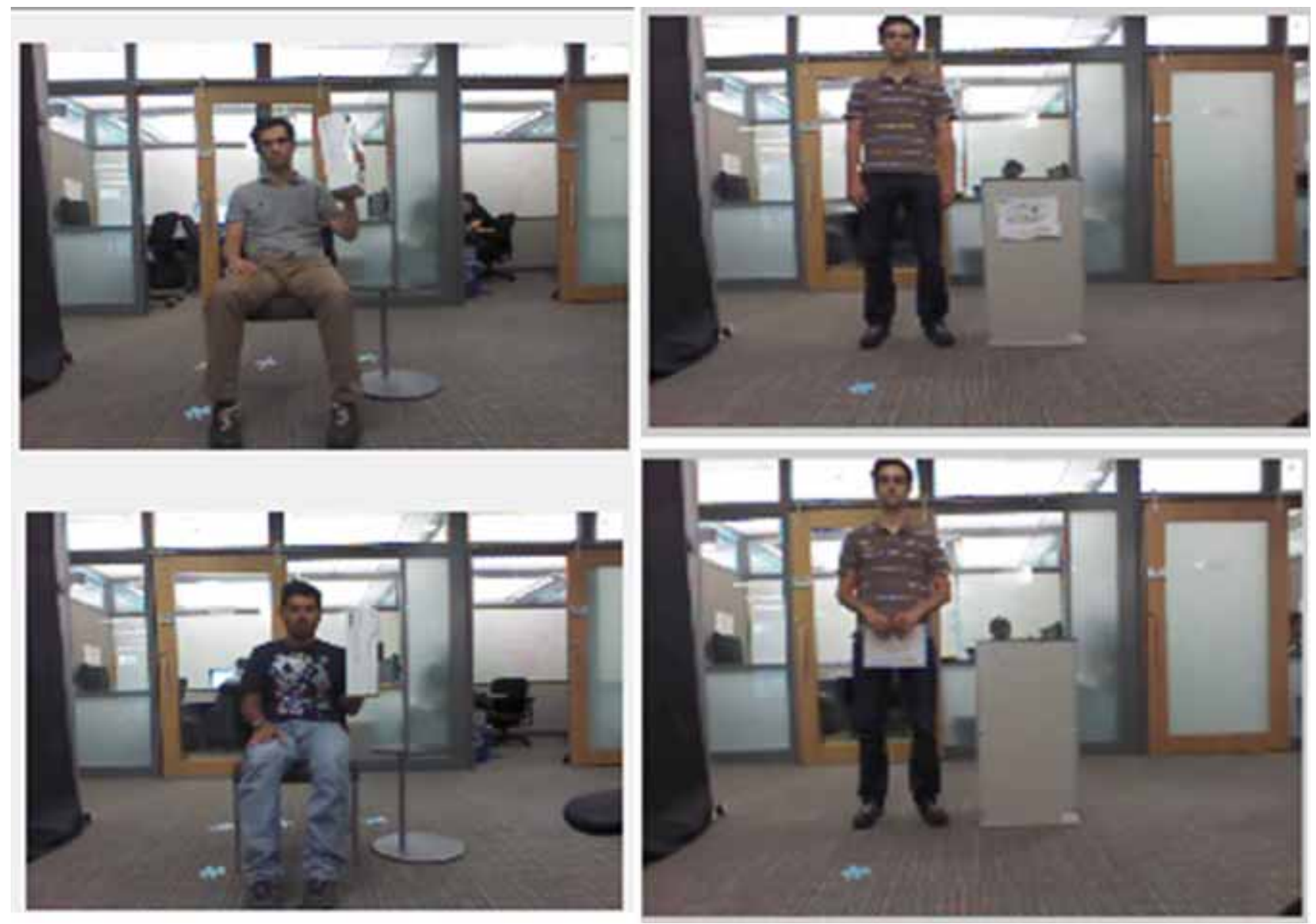

Figure 1. MSR action dataset. 


\section{Overview of content-based video retrieval}

The main challenge in a CBVR system remains in bridging the semantic gap. This is because low-level features are measured easily whereas the high-level feature retrieval process from the user query is difficult. Aim of CBVR is translating a question queried by a human and retrieval of results in terms of both feature levels. Tasks performed by CBVR system are classified into three steps: video segmentation, key frame extraction and video indexing (see figure 2).

\subsection{Shot segmentation}

The first step of video browsing and retrieval is dividing the video sequences into shots. A shot is defined as an image sequence that represents continuous action that is captured from single cameras. These shots are joined together in the video production for forming a complete sequence. Shots are a small indexing unit that can perceive high-level concepts by combining inter- and intra-shot relationships. Shot segmentation is of two types: discontinuous and continuous transitions that include editing and special effects. Instead of referring to each video frame, shots that represent video frames can be easier to construct.

\subsection{Key frame extraction}

Key frames provide a suitable framework for browsing and retrieval. As mentioned earlier the common way of representing video segments is by shot sequences of key frames. In general, meaningful frames contain main contents of the shot. This method is helpful when a user searches for a video content. When a video searching is initiated the relevant content or image is compared to key frames using similarity distance measurement. Many authors have proposed several automated ways for key frame extraction. One such process is clustering. Clustering is used in various disciplines such as pattern recognition and information retrieval.

\subsection{Feature extraction}

Extraction of information from a frame is called as feature extraction. Frames are composed of edges and shapes of grey level. Segmentation involves separating number of

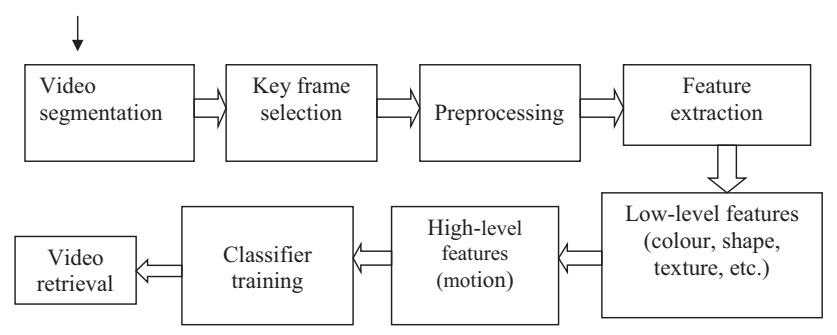

Figure 2. Content-based video retrieval process. features of interest taken one at a time. In our approach the low-level features such as shape, colour and texture are retrieved from the frames. Detailed process of feature extraction is discussed in following sections.

2.3a Visual low-level feature extraction: Features namely colour histogram, edge histogram $(\mathrm{EH})$ and edge direction histogram are extracted by sobel operator and classification is done through SVM classifier.

- Colour histogram: In case of recognition using shape or motion, colour information is not needed. Colour images are converted to grey scale and also intensity values of frames are calculated for computation reasons. Here we have used colour present in frames for processing. Colour histogram is obtained for each class of frames. It is useful in finding colour distribution of pixels in image. Colour is not uniform over all the frames; hence we have transformed the frames to colour spaces. Colour histogram computes the variance in colour for given frame. These frames are then converted from RGB colour space to $\mathrm{HSV}$ and $\mathrm{YcbCr}$ colour spaces since variance in colour becomes observable. Here $H$ represents the hue that describes wavelength of colour.

\section{Algorithm \\ Input: $R G B$ colour image frame of a video \\ Output: Colour histogram \\ Begin}

- Read the input image in RGB space

- Convert $R G B$ in to $H S V$ and $Y C_{b} C_{r}$ colour space

- Extract Hue(H), Saturation(S), Luminance(Y) and Intensity Value ( $V)$

- Use the extracted Hue, Intensity and Saturation to obtain histogram

- Similarly Luminance, Chroma components are used to obtain colour histogram

- Normalize the colour histogram

End

- Edge histogram texture feature: $\mathrm{EH}$ is used for extracting the textural feature of image. It is used in characterization of image boundaries and hence it is considered as an important stage in feature extraction. $\mathrm{EH}$ is used for segmentation of object from background. As mentioned earlier, at first the image in RGB space is converted to $\mathrm{YcbCr}$ colour space and colour histograms are obtained. Filter coefficients are (see figure 3) used as edge filters in five directions. The EH is obtained by computing rate of change of image at coordinates $(x, y)$ in all the five directions with a threshold value of 100 as given in Eq. (1). We have also applied different thresholds on the same image; when a lower threshold is used, gentle edges are detected and when the threshold increases more finite 


$$
\left(\begin{array}{rr}
1 & -1 \\
1 & -1
\end{array}\right)\left(\begin{array}{rr}
1 & 1 \\
-1 & -1
\end{array}\right)\left(\begin{array}{cc}
\sqrt{2} & 0 \\
0 & -\sqrt{2}
\end{array}\right)\left(\begin{array}{lr}
0 & \sqrt{2} \\
-\sqrt{2} & 0
\end{array}\right)\left(\begin{array}{rr}
2 & -2 \\
-2 & 2
\end{array}\right)
$$

Figure 3. (a) Horizontal edge filter, (b) vertical edge filter, (c) diagonal $\left(45^{\circ}\right)$ edge filter, (d) diagonal $\left(135^{\circ}\right)$ edge filter and (e) non-diagonal (isotropic) edge filter.

edges are detected. Gentle-edge pixels give a low grey scale value and higher value is given for sharper edges.

$$
\theta=\arctan \left[\frac{V_{y}}{V_{x}}\right]
$$

where $V_{y}$ and $V_{x}$ are gradient vectors, respectively, in $y$ and $x$ directions.

Sobel operator is used in calculating edges and direction of edges. The histogram represents the frequencies of occurrences of five classes of edges for a given image. Histogram is calculated for each image and edge mask is formed with a threshold value of 100. Edge pixels are obtained by the gradients whose magnitude is greater than 100. The sobel filter captures the spatial distribution of edges in six directions with a filter mask. This has eight bins corresponding to sobel filters for counting number of edge pixels in eight directions [9].

Algorithm
Input: RGB colour image of a video frame
Output: Edge histogram and edge direction histogram of
plant image
Begin
- Get RGB image of given frame
- Convert it to YcbCr colour space
- Extract luminance of image
- Using filter mask coefficients obtain edge and edge
- direction histogram
- Compute gradient in respective direction of filter mask
- If(gradient value $>$ threshold)
- Draw edge map
- Normalize edge histogram and edge direction
histogram

End

- Shape feature extraction: Shape is a primitive feature for image description. For the filtration of shape feature the image is first converted to grey scale from RGB colour space. Craig's formula is applied for converting the RGB to grey scale image and mean filter is applied for noise removal. Let $I_{G}, I_{R}, I_{B}$, be the RGB weights of a frame; then

$$
\operatorname{greyscale}\left(I_{g}\right)=0.2989 I_{R}+0.5870 I_{G}+0.1140 I_{B}
$$

Spatial filtering is applied on each individual pixel of a square or rectangular window surrounding each pixel in frame. This filtered image is then clustered to identify pixels that have same grey levels, colour and textures. Clustering groups objects of different features. $K$-means clustering technique is used here. The algorithm for shape feature extraction is as follows.

\section{Algorithm}

- Select $K$ data items from input as initial centroids

- Assign remaining data items that are apart from selected initial centroids to cluster $K$ which has the closest centroid.

- Calculate new centroid until convergence occurs.

- As a result the clustered regions of de-noised image is identified

The $\mathrm{K}$ regions are blurred to remove noises. Then the edges with greater magnitudes are marked. Hence the edges are identified and smoothed for sharpening to remove a number of connected components that are considered as unnecessary in clustered regions. Therefore the diverse shapes in the frame are extracted and thus shape feature is retrieved.

- Low-level feature classification using SVM: SVM achieves state-of-art performance in real-world applications and works well on unseen data. Unlike neural networks SVM produces reproducible results. Model generalization performance is increased by the ease of computation of error bounds. SVM works by mapping input vector to a high-dimension feature space and finds an optimal hyper-plane that separates classes by maximizing margin [10]. This selects only a subset of training data points called as support vectors. Mapping the input space to the feature space is done by a kernel trick that provides mapping to a high dimension without the need to specifically visit the place. Radial basis kernel and linear kernel are among the most widely used kernels in SVM. These were designed as binary classifiers; hence various techniques are used to extend SVM to a multi-class problem.

The equation of SVM that is used in estimating the decision function is given as follows:

$$
f(x)=\operatorname{sign}\left(\sum_{n=1}^{l} y_{n} \propto_{n} k\left(x, x_{n}+b\right)\right) .
$$

Here $l$ is the number of support vectors, $b$ refers to the bias term, $y_{n} \in\{-1,+1\}$ is the class sign of support vector to which it belongs and solution of $\propto$ is obtained by the following quadratic optimization problem: 


$$
\begin{gathered}
\min \frac{1}{2} w^{T} w+C \sum_{i=1}^{p} \varepsilon_{i} \\
\text { s.t. } y_{i}\left(w^{T} \theta\left(x_{i}\right)+b\right) \geq 1-\varepsilon_{i} \\
\epsilon_{i} \geq 0, \quad i=1, \ldots, p
\end{gathered}
$$

Number of support vectors cannot be greater than the number of data points in the dataset. SVM classifier is trained with 256 colour distribution values for each colour space, HSV and YcbCr. Here the SVM classifier is constructed with 256 training samples and 3 output classes. This uses a decision function to group 256 input training samples into three class labels. These labels are generated with respect to number of support vectors. Colour histogram of images spreads between 0 and 255. Classification accuracy is improved with edge information of images that belong to the same video. Hence the edge texture has proved to be a more discriminating feature for classification. Edge texture features are suitable when the same background colour images are taken for consideration. A limitation of SVM is that it lacks the ability of handling dynamic systems. This can be addressed by converting the features to fixed long vectors before applying to the SVM. However, this results in long vectors, thus affecting the performance.

2.3b Visual semantic features: Combination of several concepts such as objects, scenes and human actions forms an event. Earlier works have adopted many promising strategies for event detection. This can be categorized into tracking, flow, spatio-temporal shapes and interest points. Methods based on the tracking process handle the video frame-by-frame and segment an object of interest from background by matching the current frame against the model. A trace of model parameters is generated by following the object motion through time and this can be compared with target spatio-temporal pattern for determining the observed event that is of interest. Flow-based methods act directly on spatio-temporal sequence to recognize the specified pattern by brute-force correlation without segmentation. In shape-based methods the spatiotemporal volume of video sequences are considered as a 3D object. The goal of shape-based methods is to recognize an event by shape. Space-time interest points suffer drawbacks in 2D analogues such as failure to capture smooth motion and tendency to generate spurious detections at boundaries [7].

- Deep learning: This is different from traditional object detection systems that design manually deep models able to jointly optimize all the layers and therefore the performance can be boosted. Some components are proved to be crucial for detection systems; however they may not have corresponding layers in existing deep models. Here we have used an echo state neural network for classification of scores obtained by all the layers. Compared with conventional colour images, depth maps have several advantages. Depth maps reflect pure geometry and shape cues, which are more discriminative than colour and texture including segmentation, event detection and activity recognition. Also depth images are insensitive to changes in lighting conditions. For object detection the shape of an object can be described using normal vectors in depth images instead of colour images.

- High-level feature classification using ESN: Echo state networks (ESN) are a supervised learning technique and follow the principle of recurrent neural networks (RNN). The main property of ESN is the echo state, which is described as following the network and forgetting the initial states provided by external input. This has proven to be a state of art performance in various machine learning tasks. ESN have been applied in black-box system identification. This was tested on spoken digits with noisy conditions and competitive performances when compared to the previous model proposed [11]. ESN are combined with competitive state machine framework for creating a predictive ESN classifier. It is also shown that the model was more noise robust when compared with the hidden markov model. This has proved to be an attractive classification engine when applied for automatic speech recognition. ESN start with the stems from weights of randomly assigned recurrent nodes known as reservoir and weights are learnt only in the output layer using linear read-out function.

- Architecture of ESN: The reservoir size determines the ESN's performance. Other parameters such as connectivity, spectral radius, output activation functions, shift and scale also help in achieving considering performance. ESN connections are shown in figure 4. During the training and testing the activations of reservoir and output neurons are calculated using supervised or forced supervising learning algorithms.

ESN for supervised learning: A learning system observes training dataset from feature of instances with labels as pair

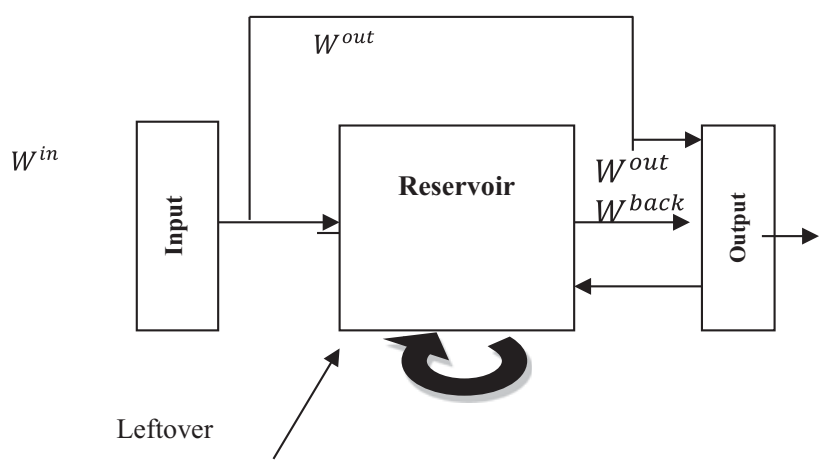

Figure 4. Echo state network. 
and is represented by $\left\{\left(x_{1}, d_{1}\right), \ldots,\left(x_{n}, d_{n}\right)\right\}$. The aim of learning algorithm is to predict output $y$ for any given feature of input $x$. In supervised learning mode the activations of ESN reservoir and output neurons are calculated using Eqs. (7) and (8):

$$
\begin{aligned}
x(n+1)= & \tanh \left(W^{i n} u(n+1)+W x(n)+W^{\text {back }} y(n)\right) \\
& + \text { leftover }
\end{aligned}
$$

where $x(n+1)$ is the reservoir step for time $(n+1)$, $u(n+1)$ is the input vector and $y(n)$ is the output obtained for supervised learning for time step $(n)$ and leftover is calculated as follows:

$$
\text { leftover }=x(n)\left(1-d_{\text {leakrate }}\right)
$$

The output $y(n+1)$ is given by

$$
y(n+1)=\tanh \left(W^{\text {out }} * x(n+1)+W^{\text {out }} * u(n+1)\right)
$$

where $y(n+1)$ is the calculated ouput for time step $(n+1)$. Weight vectors $W^{\text {in }}, W, W^{\text {back }}$ and $W^{\text {out }}$ are generated with random values between -1 and 1 initially with a connectivity parameter between 0 and 1 to generate random connections between neurons. The videos are first divided into segments and segment-level features are used to train ESN. The trained ESN computes the motion state distribution for each segment. The feature vectors extracted consist of depth images for each frame. The training video in dataset is used to train the ESN. Since not all the videos have motion we choose the videos with the highest energy. The ESN has an output with different motion states: "Pick up a box", "Lift a box/place a box", "Push/pull a chair", "Wear a hat/take off a hat", "Put on a backup/take off a backpack", and "Stand in different directions". The SVM data points are used to identify the matched frames obtained from the low-level feature extraction. The number of frames produced by SVM varies dynamically and is used for classification of motion feature.

\subsection{Video indexing and retrieval}

Video indexing is a process of tagging and organizing videos in an effective manner for fast access and retrieval. Conventional features used in the video indexing consist of colour, shape, motion, object, etc. For a better accuracy more number of features have to be used for data representation. However, as the vector dimension increases complexity of the system may increase. Hence it is essential to have minimal features for video representation. In this paper video indexing and retrieval is done with the following features: colour, texture, shape and motion. Text-based video indexing and retrieval is the current research topic. This works by expanding the semantics of a query and using the glimpse matching method that performs approximate matching instead of exact matching. The difficulty of processing highlevel queries arises from external knowledge with the description of low-level features, known as the semantic gap. A detailed view of video indexing and retrieval is discussed in the following sections.

\section{Video indexing}

\subsection{Fusion of low-level and high-level semantics}

Here video indexing is considered as a pattern recognition problem. That is, for a given pattern that describes a shot $S$, the aim of the system is to obtain high-level semantic concept $H$. For obtaining a pattern, representation from multimodal video feature extraction is done. Here we have fused the low-level and high-level feature extraction types that extract various features for accurate video classification. The general scheme for fusion algorithm is given in figure 5 .

Colour and texture features are filtered from raw dataset, the results are concatenated and classification is done through SVM classifier. Once the SVM classifier labels the key frame as matched instances, it is further investigated for motion feature detection, which then produces the final result as matched or non-matched instances. As this algorithm is a multi-stage classifier the frames that are identified as matched are extracted for deep images and classification is done via ESN. The flowchart of proposed approach is shown in figure 6.

\section{Experiment}

To evaluate our approach we have taken a real-world application task: multimedia event detection. Event detection finds an important role in video indexing and retrieval. Current event detection mechanisms aim at abnormal event detection in news and sports or abnormality in surveillance videos. In this section we evaluate the classification and fusion model on Microsoft Research (MSR) Dataset. This algorithm is based on [2] in which histograms are used for the shape and motion detection. Here we have extended the work by extracting colour and texture features and using it for classifying the dataset. In order to emphasize the

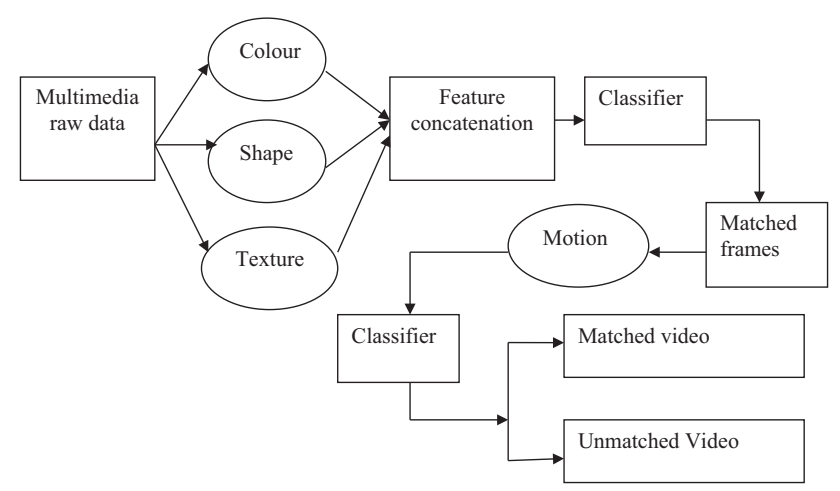

Figure 5. Representation of fusion scheme. 


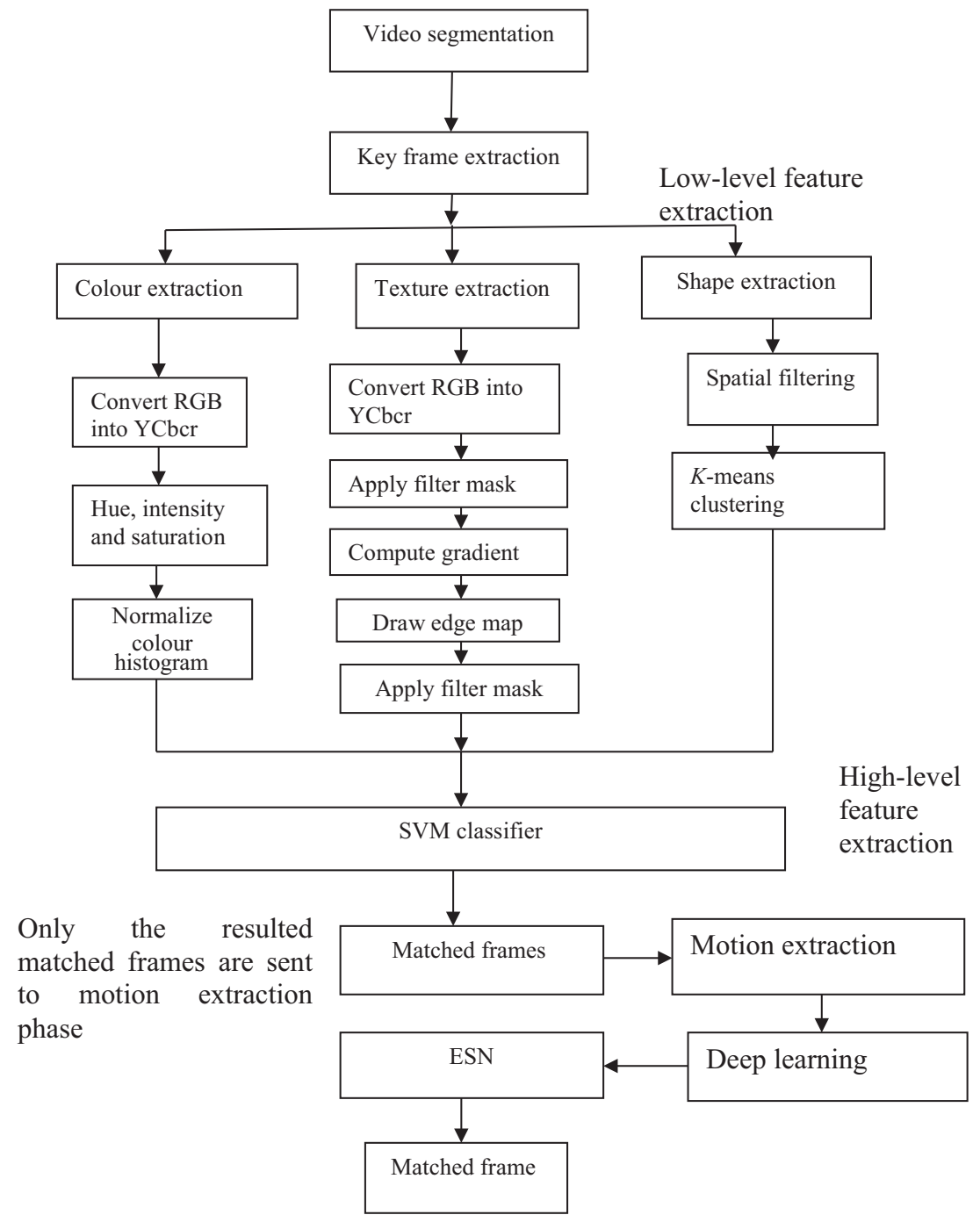

Figure 6. Flowchart of SVM-ESN.

performance of the model we have presented a fusion of both low-level and high-level feature algorithms.

\subsection{Dataset and evaluation metrics}

MSR action pair dataset consists of pairs of activities such that within each pair, motion and shape cues are similar, but correlations vary. For example actions such as "Pick up" and "Put down" consist of similar motion and shape; however the co-occurrence of object shape and hand motion is in different spatio-temporal order as shown in the figure. This dataset is useful to evaluate efficiency of descriptors to capture the prominent cues jointly in the sequence. Here we have collected six pairs of action pairs such as "Pick up a box", "Lift a box/place a box", "Push/ pull a chair", "Wear a hat/take off a hat", "Put on a backup/take off a backpack" and "Stand in different directions". These actions were performed by 10 actors three times, in which five actors were used for testing and the rest for training [12]. A Matlab code was used for the implementation of SVM shape and motion detection algorithm. Performance is calculated with the following metrics.

Accuracy

$=\frac{\text { total number of frames }- \text { number of mismatched frames }}{\text { total number of frames }}$

Precision $=\frac{\text { total number of retrival of relevant frames }}{\text { total number of relevant frames }}$

Recall $=\frac{\text { total number of retrieval of relevant frames }}{\text { total number of relevant images }}$. 


\section{Results and discussion}

Result of the experiment conducted is to be presented in this section. In addition to selection of system parameters, the parameters that are used in the final model are specified to ensure the reproducibility of the reported result. A comparison of the proposed approach and two state-of-art techniques reported in literature is included. The properties in which the model achieves higher performance is given for further improvement of the model. Here parameter selection lies under the umbrella of selection phase of the model and techniques vary in sensitivity changes of hyper-parameters. RNN are sensitive to weight initialization, which limits the ability to reproduce results with the same architecture. On the other hand SVM is more robust to changes in initial weights and reproducibility is more likely when kernel type is fixed

Table 1. Parameters.

\begin{tabular}{lc}
\hline Parameters & Values \\
\hline Reservoir size & 900 \\
Leakage rate & 0.005 \\
Scaling constant & 1.75 \\
Regularization parameter & 1000 \\
Kernel (gamma) & 0.001 \\
\hline
\end{tabular}
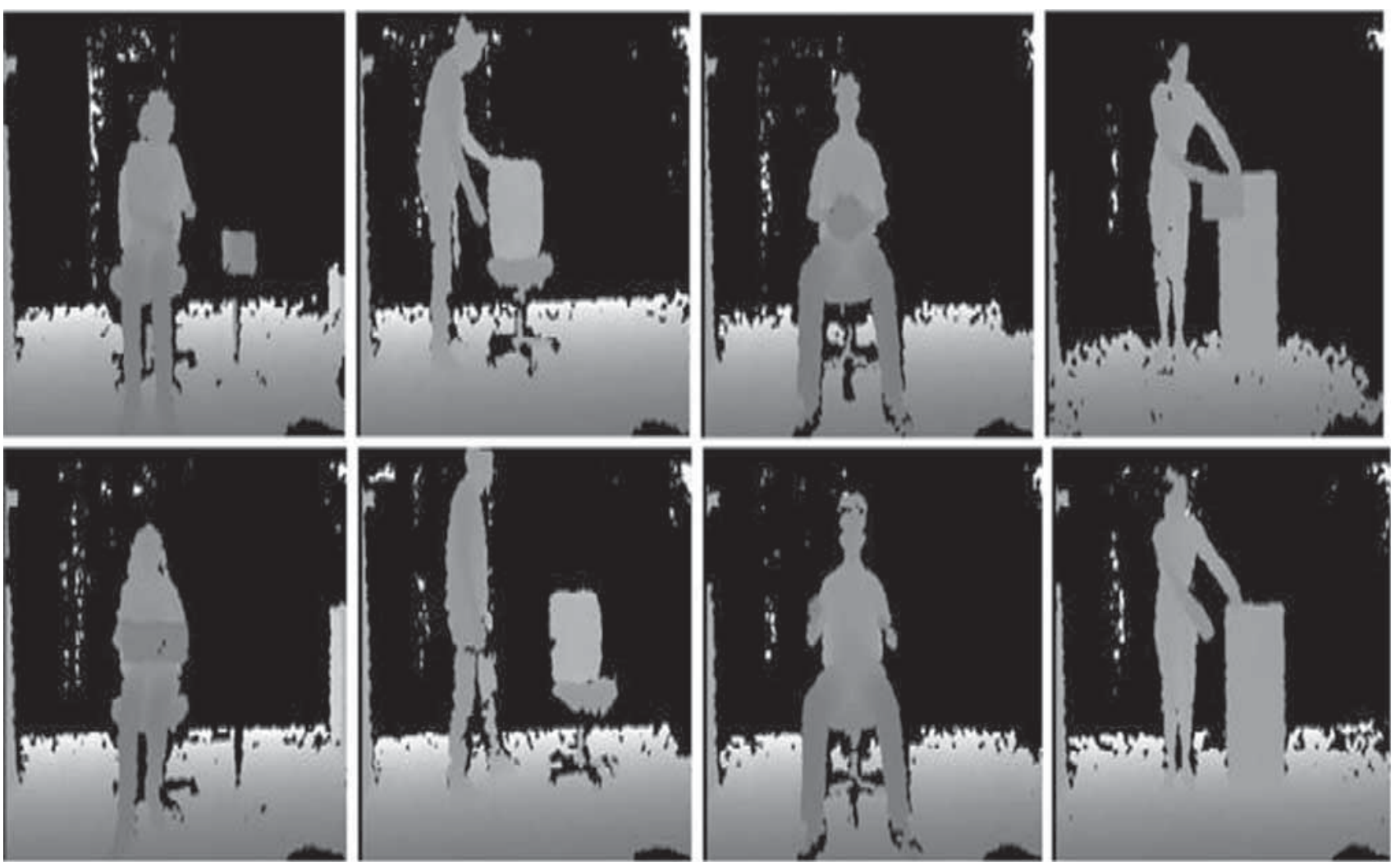

Figure 7. Depth sequences obtained from frames. 
parameters in the ESN are reservoir size and leakage rate. Using a large value for reservoir may result in high variances that are addressed by appropriate regularization technique. In our experiments we have used leakage rate larger than 0.4. Scaling constant is another hyper-parameter which is used to obtain the desired dynamic of reservoir. Parameters used in experiments are listed in table 1.

Combinations of performance of SVM and ESN are superior to the results obtained when compared with

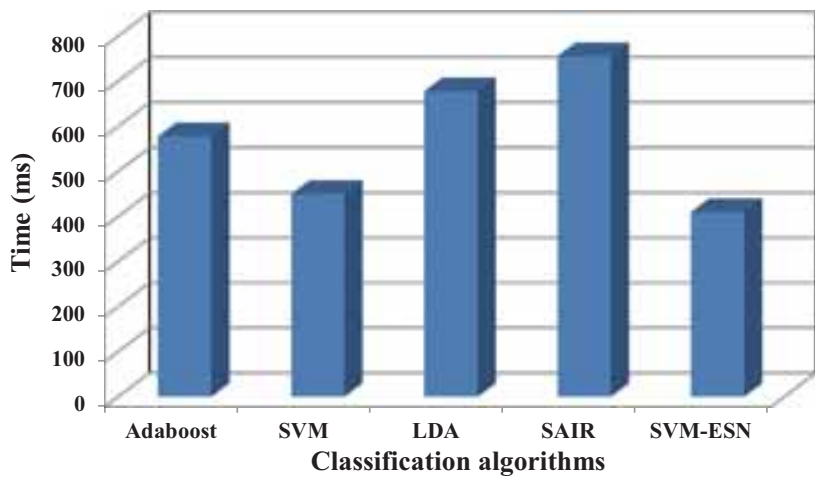

Figure 9. Comparison of execution time among different classification algorithms.
GMM-SVM. Figure 8 shows the robustness of the two approaches compared with the other. Comparison is validated using the same dataset over all the datasets. From the graph it is clear that the ESN-SVM maintains higher accuracy rate when compared with ESN and the reason behind is using a small reservoir size. The output of experiments at each stage is presented here. The depth images extracted are shown in figure 7.

As stated already we have used a action pairs dataset; each action is represented as pairs for four actions namely "Pick up a box", "Lift a box/place a box", "Push/pull a chair", "Wear a hat/take off a hat" and "Put on a backup/take off a backpack". Figure 9 shows the time taken for different classification algorithms when compared to SVMESN. It is observed from the figure that SVM-ESN takes less time than other algorithms. The user interface for experiments is shown in figure 10, in which the training and testing videos are fed into the system to predict the matched event.

Table 2 shows the detection rate of different algorithms for each event and table 3 gives comparison of the algorithms in terms of precision, recall and accuracy percentage.

Based on the results obtained the proposed approach can improve the system performance. Using a reservoir from

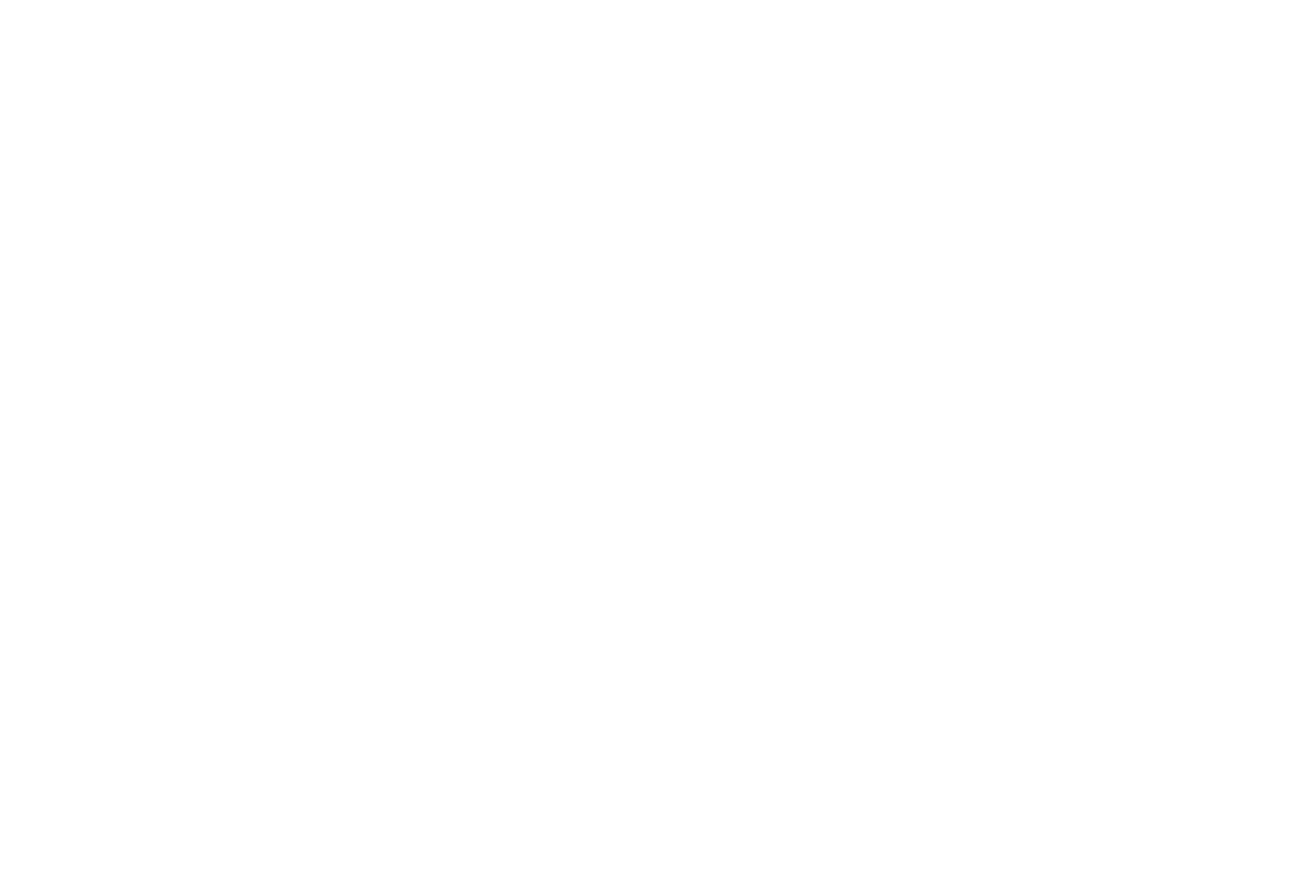

Figure 10. Detection of matched event with training and testing video. 
Table 2. Comparison of algorithm accuracy for different algorithms.

\begin{tabular}{|c|c|c|c|c|c|}
\hline \multirow[b]{2}{*}{ Event description } & \multicolumn{5}{|c|}{ Algorithms } \\
\hline & Adaboost & SVM & LDA & SAIR & SVM-ESN \\
\hline Pick up a box & 72.45 & 78.54 & 67.43 & 76.43 & 80.34 \\
\hline Lift/place a box & 56.32 & 82.45 & 75.34 & 57.76 & 83.67 \\
\hline Push/pull a chair & 73.34 & 81.98 & 87.52 & 78.54 & 92.65 \\
\hline Wear hat/take off a hat & 68.54 & 76.93 & 73.23 & 89.45 & 83.17 \\
\hline Put on/take off a backpack & 78.34 & 73.90 & 65.32 & 78.65 & 95.32 \\
\hline
\end{tabular}

Table 3. Overall comparison in terms of precision, recall and accuracy percentage.

\begin{tabular}{lccc}
\hline Algorithms & Precision & Recall & Accuracy percentage \\
\hline Adaboost & 67.9 & 63.65 & 78.93 \\
SVM & 78.3 & 75.23 & 80.54 \\
LDA & 56.3 & 67.1 & 83.32 \\
SAIR & 84.3 & 78.4 & 86.43 \\
SVM-ESN & 90.8 & 88.43 & 91.2 \\
\hline
\end{tabular}

small to medium size yields higher accuracy rate. With easily computed error bounds of SVM, the SVM-ESN combination achieves robust performance. On applying the SVM-ESN classifier to multiclass problems it needs more time; this is due to the fact that SVM is a binary classifier that requires constructing at least equal to the number of problem classes. Hence SVM-ESN is limited to multi-class classification problems with large or medium number of classes.

\section{Conclusion}

In this paper we have proposed a multi-class SVM-ESN classifier approach to bridge the semantic gap between lowlevel and high-level features. Event detection is done using two stages: initially the low-level features such as colour and texture are extracted using colour histogram and edge detection for training video. The classification of the extracted features is done using SVM. In the second stage, deep learning is done for the frames that are labelled as matched by the SVM classifier. Depth images are classified using ESN.

To evaluate the performance of our proposed approach we conducted experiments on the MSR 3D action pair dataset. This dataset is publicly available and contains pairs of actions performed by different humans. The results obtained are then compared with ESN and GMMSVM models. The SVM-ESN attains a high accuracy rate of $96.66 \%$, which shows the effect of multi-stage SVM-ESN classifier in classification problems such as human activity recognition. This method is suitable when the reservoir size is small. Future work can be extended by using various optimization techniques with ESN and comparing the performance. Speeding up the ESN and SVM training are important for large-scale event detection.

\section{References}

[1] Hoi S C H and Lyu M R 2007 A multimodal and multilevel ranking framework for content-based video retrieval. In: Proceedings of the IEEE International Conference on Acoustics, Speech and Signal Processing, 2007 (ICASSP 2007), vol. 4

[2] Kamishima Y, Nakamasa I and Koichi S 2013 Event detection in consumer videos using GMM supervectors and SVMs. EURASIP J. Image Video Process. 1: 1-13

[3] Lu S, King I and Lyu M R 2004 Video summarization by video structure analysis and graph optimization. In: Proceedings of the 2004 IEEE International Conference on Multimedia and Expo, ICME'04, vol. 3

[4] Assfalg J et al 2002 Soccer highlights detection and recognition using HMMs. Proceedings of ICME (1), pp. 825-828

[5] Adam A et al 2008 Robust real-time unusual event detection using multiple fixed-location monitors. IEEE Trans. Pattern Anal. Mach. Intell. 30(3): 555-560

[6] Gupta S, Gupta N and Kumar S 2011 Evaluation of object based video retrieval using SIFT. Int. J. Soft Comput. Eng. ISSN: 2231-2307

[7] Ma Z et al 2013 Multimedia event detection using a classifier-specific intermediate representation. IEEE Trans. Multimedia 15(7): 1628-1637

[8] Anami B S, Suvarna S N Govardhan A 2010 A combined color, texture and edge features based approach for identification and classification of Indian medicinal plants. Int. J. Comput. Appl. 6(12): 45-51

[9] Vapnik V N 1999 An overview of statistical learning theory. IEEE Trans. Neural Netw. 10(5): 988-999

[10] Schrauwen B and Buesing L 2009 A hierarchy of recurrent networks for speech recognition. In: Proceedings of the Workshop on Deep Learning for Speech Recognition and Related Applications

[11] Delezoide B 2006 Multimedia movie segmentation using low-level and semantic features. Ph.D. Thesis, Université Pierre et Marie Curie, Paris

[12] Oreifej O and Liu Z 2013 Hon4d: histogram of oriented 4d normals for activity recognition from depth sequences. In: Proceedings of the IEEE Conference on Computer Vision and Pattern Recognition (CVPR) 